\title{
On the Bivariate Nakagami-Lognormal Distribution and Its Correlation Properties
}

\author{
Juan Reig, Lorenzo Rubio, and Vicent M. Rodrigo-Peñarrocha \\ Grupo de Radiación Electromagnètica (GRE), Universitat Politècnica de València, Camino de Vera s/n, 46022 Valencia, Spain \\ Correspondence should be addressed to Juan Reig; jreigp@dcom.upv.es
}

Received 9 May 2014; Accepted 27 June 2014; Published 18 August 2014

Academic Editor: Jose F. Paris

Copyright (C) 2014 Juan Reig et al. This is an open access article distributed under the Creative Commons Attribution License, which permits unrestricted use, distribution, and reproduction in any medium, provided the original work is properly cited.

\begin{abstract}
The bivariate Nakagami-lognormal distribution used to model the composite fast fading and shadowing has been examined exhaustively. In particular, we have derived the joint probability density function, the cross-moments, and the correlation coefficient in power terms. Also, two procedures to generate two correlated Nakagami-lognormal random variables are described. These procedures can be used to evaluate the robustness of the sample correlation coefficient distribution in both macro- and microdiversity scenarios. It is shown that the bias and the standard deviation of this sample correlation coefficient are substantially high for large shadowing standard deviations found in wireless communication measurements, even if the number of observations is considerable.
\end{abstract}

\section{Introduction}

In wireless communications, the received signal is subjected to fading due to two physical mechanisms. On one hand, the multipath components cause rapid and deep fading in displacements of few wavelengths (small-scale area). This is the well-known short-term fading or fast fading, which has been extensively analyzed in the literature $[1,2]$. This fast fading has been modeled statistically using the Rice, Rayleigh, Nakagami- $m$, and Weibull distributions. The Nakagami- $m$ distribution is frequently employed to model the fast fading since it fits better than the other distributions in many measurement campaigns [3,4]. On the other hand, the received signal fluctuates slowly around a mean in displacements of hundreds of wavelengths (large-scale area). This variation is known as long-term fading or shadowing. This shadowing is due to the temporal blockage of the direct component between the transmitter and receiver terminals. The shadowing is commonly modeled statistically by a lognormal distribution. Values of standard deviations for shadowing reported in the literature range from 5 to $12 \mathrm{~dB}$ in macrocells, from 6 to $10 \mathrm{~dB}$ in microcells, and from 3 to $10 \mathrm{~dB}$ in indoor environments [5].

In the analysis of wireless propagation channels, these components are traditionally separated to model both the fast fading and shadowing. Nevertheless, this separation is troublesome in scenarios as in indoor and vehicular channels, where the variation of the received signals is considerably high, with few displacements in terms of the wavelength, and thus these effects are severely overlapped.

Therefore, several distributions have been proposed to describe the composite fast fading and shadowing. Initially, the Rayleigh-lognormal distribution was used by Suzuki to model this compound fading [6]. Also, the Nakagamilognormal distribution has been employed to model the composite fading [7-9]. Recently, some other distributions as the $\alpha-\mu$ [10], the generalized- $K$ [11], or the mixture gamma (MG) distribution [12] have been proposed in the literature. Nevertheless, the results derived from a measurement campaign carried out in a macrocellular urban environment [13] have shown that the Nakagami-lognormal is the bestfit distribution compared with the Rayleigh-lognormal, $\alpha-\mu$, and generalized- $K$ distributions.

The effect of the correlated shadowing or correlated fast fading on the performance parameters in wireless communication diversity has attracted the attention of several researches [14-19]. Nevertheless, all the above works have assumed either correlated fast fading with independent shadowing or correlated shadowing with independent fast fading. 
Therefore, to the best of the authors' knowledge an analysis of the general bivariate distribution Nakagami-lognormal with correlated both fading and shadowing and arbitrary fading parameters is novel in the literature.

Thus, in this paper the joint probability density function (PDF) and the cross-moments of the bivariate Nakagamilognormal distribution are derived. Also, we have focused our attention on calculating the correlation coefficient between the composite signals. This correlation coefficient is particularized for two wireless scenarios of interest: macro- and microdiversity cases.

Two procedures to generate two correlated Nakagamilognormal random variables (RVs) are proposed and several examples for the sample correlation coefficient are evaluated in both macro- and microdiversity scenarios.

This paper is organized as follows. First, the joint PDF, the central moments, and the correlation coefficient of the Nakagami-lognormal distribution are derived in Section 2. Second, in Section 3 we describe two procedures for generating Nakagami-lognormal RVs and several results are analyzed. Finally, the conclusions are discussed in Section 4.

\section{Bivariate Nakagami-Lognormal Distribution}

2.1. Joint Probability Density Function. Let $r_{f_{1}}$ and $r_{f_{2}}$ be correlated Nakagami- $m$ RVs, representing fast fading processes with the same mean power equal to unity, whose marginal PDFs are given by

$$
\begin{array}{r}
p_{r_{f_{j}}}\left(r_{f_{j}}\right)=\frac{2}{\Gamma\left(m_{j}\right)} m_{j}^{m_{j}} r_{f_{j}}^{2 m_{j}-1} \exp \left(-m_{j} r_{f_{j}}^{2}\right), \\
r_{f_{j}} \geq 0, \quad j=1,2,
\end{array}
$$

where $m_{j}$ is the fading parameter and $\Gamma(z)=\int_{0}^{\infty} t^{z-1} e^{-t} d t$ is the Gamma function [20, (6.1.1)].

The correlation coefficient between $r_{f_{1}}$ and $r_{f_{2}}$ in power terms can be expressed as

$$
\rho_{f}=\frac{E\left(r_{f_{1}}^{2} r_{f_{2}}^{2}\right)-E\left(r_{f_{1}}^{2}\right) E\left(r_{f_{2}}^{2}\right)}{\sqrt{\operatorname{var}\left(r_{f_{1}}^{2}\right) \operatorname{var}\left(r_{f_{2}}^{2}\right)}},
$$

where $\operatorname{var}(\cdot)$ denotes the variance.

We can define $r_{s_{1}}$ and $r_{s_{2}}$ as correlated lognormal RVs corresponding to shadowing processes, whose marginal PDFs can be written as

$$
\begin{array}{r}
p_{r_{s_{j}}}\left(r_{s_{j}}\right)=\frac{1}{\sqrt{2 \pi} \sigma_{j} r_{s_{j}}} \exp \left(-\frac{\left(\ln \left(r_{s_{j}}\right)-\mu_{j}\right)^{2}}{2 \sigma_{j}^{2}}\right), \\
r_{s_{j}} \geq 0, \quad j=1,2,
\end{array}
$$

where

$$
\begin{aligned}
& \mu_{j}=\frac{\ln (10)}{10} \mu_{d j}, \\
& \sigma_{j}=\frac{\ln (10)}{10} \sigma_{d j},
\end{aligned}
$$

$\mu_{d j}$ being the mean of the associated Gaussian process of the shadowing, expressed in $\mathrm{dBV} / \mathrm{m}, \sigma_{d j}$ the standard deviation of the associated normal distribution corresponding to the lognormal shadowing, expressed in $\mathrm{dB}$. The power correlation coefficient between the associated Gaussian processes, $x_{1}=20 \log r_{s_{1}}$ and $x_{2}=20 \log r_{s_{2}}$, is denoted by $\rho_{s}$ and it is defined in the same way as (2), by substituting $x_{1}$ and $x_{2}$ for $r_{f_{1}}$ and $r_{f_{2}}$, respectively.

If we calculate $r_{j}=r_{f_{j}} \cdot r_{s_{j}}, j=1,2$, then $r_{j}$ is Nakagamilognormal distributed, with PDF given by

$$
\begin{aligned}
& p_{r_{j}}\left(r_{j}\right) \\
& =\int_{0}^{\infty} \frac{2}{\Gamma\left(m_{j}\right)}\left(\frac{m_{j}}{\Omega_{j}}\right)^{m_{j}} r_{j}^{2 m_{j}-1} \exp \left(-\frac{m_{j} r_{j}^{2}}{\Omega_{j}}\right) \\
& \quad \times \frac{1}{\sqrt{2 \pi} \sigma_{j} \Omega_{j}} \exp \left(-\frac{\left(\ln \left(\Omega_{j}\right)-\mu_{j}\right)^{2}}{2 \sigma_{j}^{2}}\right) d \Omega_{j} \quad r_{j} \geq 0,
\end{aligned}
$$

where $\Omega_{j}$ is a lognormal RV which represents the shadowing process. Note that the PDF given by (6) corresponds to the Nakagami-lognormal PDF in power terms given by $[8,(8)]$.

The $n$th moment of $r_{j}, j=1,2$, is calculated as

$$
E\left(r_{j}^{n}\right)=\frac{\Gamma\left(m_{j}+n / 2\right)}{m_{j}^{n / 2} \Gamma\left(m_{j}\right)} \exp \left(\frac{n}{2} \mu_{j}+\frac{n^{2}}{8} \sigma_{j}^{2}\right),
$$

where $E(\cdot)$ denotes expectation. Note that $(7)$ is equivalent to $[21,(2.59)]$ by substituting $2 n$ instead of $n$ in (7), which corresponds to the $n$th moment of the Gamma-lognormal distribution.

Combining [22, (2.1)] and [23, (12)], the joint PDF of $r_{1}$ and $r_{2}$ can be obtained as

$$
\begin{aligned}
& p_{r_{1}, r_{2}}\left(r_{1}, r_{2}\right) \\
& =4\left(1-\delta_{f}\right)^{m_{2}} \sum_{k=0}^{\infty} \frac{\left(m_{1}\right)_{k}}{\Gamma\left(m_{1}+k\right) \Gamma\left(m_{2}+k\right) k !} \\
& \times \delta_{f}^{k} r_{1}^{2\left(m_{1}+k\right)-1} r_{2}^{2\left(m_{2}+k\right)-1} \\
& \times \int_{0}^{\infty} \int_{0}^{\infty}\left(\frac{m_{1}}{\Omega_{1}\left(1-\delta_{f}\right)}\right)^{m_{1}+k}\left(\frac{m_{2}}{\Omega_{2}\left(1-\delta_{f}\right)}\right)^{m_{2}+k} \\
& \times \exp \left[-\left(\frac{m_{1} r_{1}^{2}}{\Omega_{1}\left(1-\delta_{f}\right)}+\frac{m_{2} r_{2}^{2}}{\Omega_{2}\left(1-\delta_{f}\right)}\right)\right] \\
& \times{ }_{1} F_{1}\left(m_{2}-m_{1}, m_{2}+k ; \frac{m_{2} \delta_{f}}{\Omega_{2}\left(1-\delta_{f}\right)} r_{2}^{2}\right) \\
& \times \frac{1}{2 \pi \Omega_{1} \Omega_{2} \sigma_{1} \sigma_{2} \sqrt{1-\rho_{s}^{2}}} \exp \left(-\frac{q}{2}\right) d \Omega_{1} d \Omega_{2},
\end{aligned}
$$


being

$$
\begin{aligned}
q=\frac{1}{1-\rho_{s}^{2}}\left[\left(\frac{\ln \Omega_{1}-\mu_{1}}{\sigma_{1}}\right)^{2}+\left(\frac{\ln \Omega_{2}-\mu_{2}}{\sigma_{2}}\right)^{2}\right. \\
\\
\left.\quad-2 \rho_{s}\left(\frac{\ln \Omega_{1}-\mu_{1}}{\sigma_{1}}\right)\left(\frac{\ln \Omega_{2}-\mu_{2}}{\sigma_{2}}\right)\right],
\end{aligned}
$$

where $(a)_{b}=\Gamma(a+b) / \Gamma(a)$ is the Pochhammer symbol [20, $(6.1 .22)],{ }_{1} F_{1}(\cdot, \cdot ; \cdot)$ is the confluent hypergeometric function $[20,(13.1 .2)]$, and $\delta_{f}=\rho_{f} \sqrt{m_{2} / m_{1}}$. The Nakagami- $m$ variables in (8) are ordered to fulfill the condition $m_{2} \geq m_{1}$ [23]. Thus, the correlation coefficient between the Nakagami$m$ processes in power terms is limited to $\rho_{f}<\sqrt{m_{1} / m_{2}}$.

The joint bivariate Nakagami-lognormal PDF can be simplified for $m=m_{1}=m_{2}$ using [24, (1)], [3, (126)] as

$$
\begin{aligned}
& p_{r_{1}, r_{2}}\left(r_{1}, r_{2}\right) \\
& =\int_{0}^{\infty} \int_{0}^{\infty} \frac{4 m\left(m r_{1} r_{2}\right)^{m}}{\Gamma(m) \Omega_{1} \Omega_{2}\left(1-\rho_{f}\right)\left(\sqrt{\Omega_{1} \Omega_{2} \rho_{f}}\right)^{m-1}} \\
& \times \exp \left[-\left(\frac{m_{1} r_{1}^{2}}{\Omega_{1}\left(1-\delta_{f}\right)}+\frac{m_{2} r_{2}^{2}}{\Omega_{2}\left(1-\delta_{f}\right)}\right)\right] I_{m-1} \\
& \times\left(\frac{2 m \sqrt{\rho_{f}} r_{1} r_{2}}{\sqrt{\Omega_{1} \Omega_{2}}\left(1-\rho_{f}\right)}\right) \\
& \times \frac{1}{2 \pi \Omega_{1} \Omega_{2} \sigma_{1} \sigma_{2} \sqrt{1-\rho_{s}^{2}}} \exp \left(-\frac{q}{2}\right) d \Omega_{1} d \Omega_{2},
\end{aligned}
$$

where $I_{\nu}(\cdot)$ is the modified Bessel function of the first kind $[20,(9.6 .3)]$, and $q$ is given by (9).

Assuming $\rho_{s}=1$ in (8) and (10), the infinite summation is not convergent. In order to overcome it, we can alternatively express the joint PDF for $\rho_{s}=1, m=m_{1}=m_{2}, \mu=\mu_{1}=\mu_{2}$, and $\sigma=\sigma_{1}=\sigma_{2}$ as

$$
\begin{aligned}
p_{r_{1}, r_{2}}\left(r_{1}, r_{2}\right)= & \int_{0}^{\infty} \frac{4 m\left(m r_{1} r_{2}\right)^{m}}{\Gamma(m)\left(1-\rho_{f}\right) \rho_{f}^{(m-1) / 2} \Omega^{m+1}} \\
& \times \exp \left[-\frac{m}{\Omega\left(1-\rho_{f}\right)}\left(r_{1}^{2}+r_{2}^{2}\right)\right] \\
& \times I_{m-1}\left(\frac{2 m \sqrt{\rho_{f}} r_{1} r_{2}}{\Omega\left(1-\rho_{f}\right)}\right) \frac{1}{\sqrt{2 \pi} \sigma \Omega} \\
& \times \exp \left(-\frac{(\ln (\Omega)-\mu)^{2}}{2 \sigma^{2}}\right) d \Omega .
\end{aligned}
$$

2.2. Correlation Coefficient. Using [23, (16)] and [25, (2.1)], the cross-moments of the bivariate Nakagami-lognormal distribution can be calculated as

$$
\begin{aligned}
& E\left(r_{1}^{n} r_{2}^{p}\right) \\
& =\frac{\Gamma\left(m_{1}+n / 2\right) \Gamma\left(m_{2}+p / 2\right)}{\Gamma\left(m_{1}\right) \Gamma\left(m_{2}\right) m_{1}^{n / 2} m_{2}^{p / 2} 2} F_{1}\left(-\frac{n}{2},-\frac{p}{2} ; m_{2} ; \delta_{f}\right) \\
& \quad \times \exp \left(\frac{n}{2} \mu_{1}+\frac{p}{2} \mu_{2}+\frac{n^{2}}{8} \sigma_{1}^{2}+\frac{p^{2}}{8} \sigma_{2}^{2}+\frac{n p}{4} \rho_{s} \sigma_{1} \sigma_{2}\right),
\end{aligned}
$$

where ${ }_{2} F_{1}(\cdot, \cdot ; \cdot ; \cdot)$ is the Gauss hypergeometric function [20, (15.1.1)].

From (7) and (12), the correlation coefficient in power terms between two Nakagami-lognormal variables can be derived as

$$
\begin{aligned}
& \rho \\
& =\left(\frac{m_{2}+\rho_{f} \sqrt{m_{2} / m_{1}}}{m_{2}} \exp \left(\rho_{s} \sigma_{1} \sigma_{2}\right)-1\right) \\
& \quad \times\left(\sqrt{\left(\frac{m_{1}+1}{m_{1}} \exp \left(\sigma_{1}^{2}\right)-1\right)\left(\frac{m_{2}+1}{m_{2}} \exp \left(\sigma_{2}^{2}\right)-1\right)}\right)^{-1} .
\end{aligned}
$$

Note that this power correlation coefficient is not affected by the means of the associated Gaussian processes, $\mu_{j}, j=$ 1,2 , corresponding to the shadowing.

Next, we analyze the correlation coefficient between the composite fast fading shadowing of the received powers in two wireless communication scenarios.

Case 1 (macrodiversity). The macrodiversity is the technique used to combat the effects of the shadowing, by combining the signals received in several antennas separated by hundreds of wavelengths. For instance, in the macrodiversity scenario depicted in Figure 1, the mobile terminal is connected simultaneously to two base stations (BSs), BS1 and BS2. The gain obtained with macrodiversity in this scenario depends strongly on the cross-correlation coefficient of the received field strengths at BS1 and BS2.

The behavior of the site-to-site shadowing correlation coefficient has been extensively analyzed in the literature $[5,26,27]$. In [5], a model of the shadowing correlation has been proposed where the cross-correlation coefficient is given by

$$
\rho_{s}= \begin{cases}\sqrt{\frac{d_{1}}{d_{2}}} & 0 \leq \phi<\phi_{T}, \\ \left(\frac{\phi_{T}}{\phi}\right)^{\gamma} \sqrt{\frac{d_{1}}{d_{2}}} & \phi_{T} \leq \phi \leq \pi,\end{cases}
$$

where $d_{1}$ is the smallest of the two path lengths $d_{1}$ and $d_{2} ; \gamma$ is a parameter of adjustment which depends on the size and 


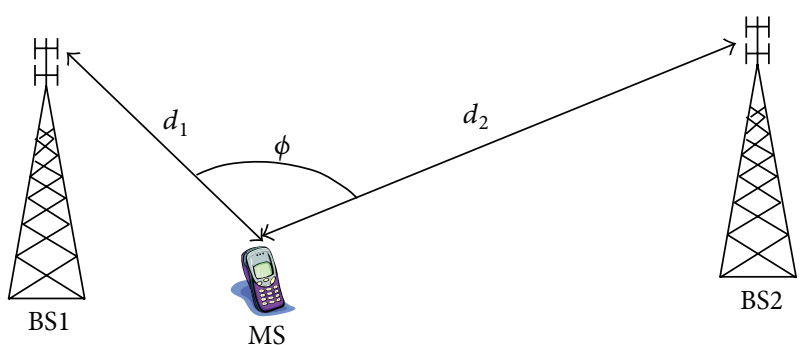

BS: base station

MS: mobile station

FIgURE 1: Cross-correlation shadowing scenario.

height of terrain and clutter, and according to the height of the BS antenna relative to them; and

$$
\phi_{T}=2 \sin ^{-1} \frac{d_{c}}{2 d_{1}}
$$

$d_{c}$ being the shadowing correlation distance.

In this situation, it is reasonable to assume that the short-term variations of the signal envelopes at BS1 and BS2 are independent, and consequently $\rho_{f}=0$. Hence, the correlation coefficient between the instantaneous powers received at BS1 and BS2 can be easily simplified from (13) as

$\rho$

$$
\begin{aligned}
= & \left(\exp \left(\rho_{s} \sigma_{1} \sigma_{2}\right)-1\right) \\
& \times\left(\sqrt{\left(\frac{m_{1}+1}{m_{1}} \exp \left(\sigma_{1}^{2}\right)-1\right)\left(\frac{m_{2}+1}{m_{2}} \exp \left(\sigma_{2}^{2}\right)-1\right)}\right)^{-1},
\end{aligned}
$$

where $\rho_{s}$ is given by (14).

Case 2 (microdiversity). The microdiversity has been extensively used to reduce the harmful effects of the fast fading. The most common methods employed in microdiversity are spatial, polarization, frequency, angular, and temporal [2]. In order to obtain a high degree of improvement using microdiversity, two conditions should be fulfilled: (i) low cross-correlation coefficients between individual diversity branches and (ii) similar mean power available from each branch of the combiner [5]. For instance, the correlation coefficient using spatial microdiversity at the BS depends basically on the antenna separation, the direction of arrival of the main contribution arriving from the mobile station (MS), and the angular spread corresponding to the scatterers surrounding the MS [28].

It is reasonable to assume the following hypotheses in a microdiversity scenario: (i) total shadowing correlation, that is, $\rho_{s}=1$; (ii) identical distributions for shadowing processes, that is, $\sigma=\sigma_{1}=\sigma_{2}$; and (iii) identical fading parameters for the short-term distributions, that is, $m=$ $m_{1}=m_{2}$. Accordingly, the correlation coefficient between the composite distributions, whose joint PDF is given by (11), is simplified from (13) to

$$
\rho=\frac{\left(m+\rho_{f}\right) \exp \left(\sigma^{2}\right)-m}{(m+1) \exp \left(\sigma^{2}\right)-m} .
$$

In this case, the correlation coefficient in power terms is limited to

$$
\frac{m\left(\exp \left(\sigma^{2}\right)-1\right)}{m\left(\exp \left(\sigma^{2}\right)-1\right)+\exp \left(\sigma^{2}\right)} \leq \rho \leq 1 .
$$

\section{Numerical Results}

In this section, we compare the values of the correlation coefficient given by (13) to those obtained by generating two correlated Nakagami-lognormal RVs using two methods.

3.1. Nakagami-Lognormal RV Generation. First, two correlated Gaussian variables will be generated following the next method. Let $x_{1}$ be a Gaussian RV with mean $\mu_{d 1}$ and standard deviation $\sigma_{d_{1}}$, denoted as $x_{1} \sim \mathcal{N}\left(\mu_{d_{1}}, \sigma_{d_{1}}\right)$. If we generate a Gaussian RV, symbolized as $y \sim$ $\mathcal{N}\left(\mu_{d_{2}}-\rho_{s}\left(\sigma_{d_{2}} / \sigma_{d_{1}}\right) \mu_{d_{1}}, \sigma_{d_{2}} \sqrt{1-\rho_{s}^{2}}\right)$, the variable $x_{2}=$ $\rho_{s}\left(\sigma_{d_{2}} / \sigma_{d_{1}}\right) x_{1}+y$ is a Gaussian RV represented as $x_{2} \sim$ $\mathcal{N}\left(\mu_{d 2}, \sigma_{d 2}\right)$, correlated $\rho_{s}$ with $x_{1}$. The pair of Gaussian correlated RVs, $x_{1}$ and $x_{2}$, are denoted as $\left\{x_{1}, x_{2}\right\} \sim$ $\mathcal{N}\left(\mu_{d 1}, \sigma_{d 1} ; \mu_{d 2}, \sigma_{d 2} ; \rho_{s}\right)$.

Second, we generate two correlated lognormal variables using the following transformations:

$$
\Omega_{1}=10^{x_{1} / 10}, \quad \Omega_{2}=10^{x_{2} / 10} .
$$

Hence, $\Omega_{1}$ and $\Omega_{2}$ are lognormal distributed, denoted as $\left\{\Omega_{1}, \Omega_{2}\right\} \sim \mathscr{L}\left(\mu_{1}, \sigma_{1} ; \mu_{2}, \sigma_{2} ; \rho_{s}\right)$. Note that the mean, the variance, and the correlation coefficient between $\Omega_{1}$ and $\Omega_{2}$ are given by Mostafa and Mahmoud [22]

$$
\begin{gathered}
E\left(\Omega_{j}\right)=\exp \left(\mu_{j}+\frac{\sigma_{j}^{2}}{2}\right), \quad j=1,2, \\
\operatorname{var}\left(\Omega_{j}\right)=\exp \left(2 \mu_{j}+\sigma_{j}^{2}\right)\left(\exp \left(\sigma_{j}^{2}\right)-1\right), \quad j=1,2, \\
\rho_{l}=\frac{\exp \left(\rho_{s} \sigma_{1} \sigma_{2}\right)-1}{\sqrt{\left(\exp \left(\sigma_{1}^{2}\right)-1\right)\left(\exp \left(\sigma_{2}^{2}\right)-1\right)}},
\end{gathered}
$$

where $\mu_{j}$ and $\sigma_{j}$ are related to the logarithmic parameters, $\mu_{d j}$ and $\sigma_{d j}$, in (4) and (5), respectively.

Finally, we generate two correlated Nakagami- $m$ variables with arbitrary fading parameters, whose mean powers are correlated lognormal RVs. These RVs, denoted as $\left\{r_{1}, r_{2}\right\} \sim$ $\mathscr{M}\left(m_{1}, \Omega_{1} ; m_{2}, \Omega_{2} ; \rho_{f}\right)$, can be generated by following the method described in [29]. Each RV, $r_{j}, j=1,2$, follows a Nakagami- $m$ distribution, whose PDF is given by

$$
p\left(r_{j}\right)=\frac{2}{\Gamma\left(m_{j}\right)}\left(\frac{m_{j}}{\Omega_{j}}\right)^{m_{j}} r_{j}^{2 m_{j}-1} \exp \left(-\frac{m_{j} r_{j}^{2}}{\Omega_{j}}\right),
$$


where $m_{j}$ is the fading parameter; $\Omega_{j}$ is the mean power calculated in this case from (19); and $\rho_{f}$ is the power correlation coefficient. Note that $m_{2} \geq m_{1}$ and $\rho_{f}<\sqrt{m_{1} / m_{2}}$. Estimated values of the fading parameter typically range from 1 to 3 . For example, a mean of 1.56 and a standard deviation of 0.34 for the estimated fading parameters were reported in [4].

To improve in computation efficiency, we can alternatively generate the two correlated Nakagami-lognormal RVs by using a logarithmic procedure, since a log Nakagami-lognormal RV can be calculated as the sum of a log Nakagami$m$ and a Gaussian RVs [13]. Therefore, first two correlated Gaussian RVs, that is, $\left\{x_{1}, x_{2}\right\} \sim \mathcal{N}\left(\mu_{d_{1}}, \sigma_{d_{1}} ; \mu_{d_{2}}, \sigma_{d_{2}} ; \rho_{s}\right)$, are calculated as previously. Second, we generate two correlated Nakagami- $m$ RVs with average powers equal to 1 , that is, $\left(v_{1}, v_{2}\right) \sim \mathscr{M}\left(m_{1}, 1 ; m_{2}, 1 ; \rho_{f}\right)$. Next, two log Nakagami- $m$ RVs are generated by calculating $\epsilon_{1}=20 \log v_{1}$ and $\epsilon_{2}=$ $20 \log v_{2}$. Finally, the two correlated Nakagami-lognormal RVs are obtained as $r_{1}=10^{\left(x_{1}+\epsilon_{1}\right) / 20}$ and $r_{2}=10^{\left(x_{2}+\epsilon_{2}\right) / 20}$.

3.2. Sample Correlation Coefficient. We have generated $N$ observations of correlated Nakagami-lognormal RVs following the methods described above. Hence, the sample coefficient correlation, $R$, is calculated. $K$ samples of the sample correlation coefficient are obtained in order to estimate the bias and the variance of $R$. The variance of $R$ depends strongly on the skewness of the marginal Nakagami-lognormal distributions as it was shown for different examples of the lognormal distribution in [30]. The skewness of the marginal Nakagami-lognormal distributions can be evaluated from (7) as

$$
\begin{gathered}
s_{j}=\frac{E\left[\left(r_{j}-\bar{r}_{j}\right)^{3}\right]}{E\left[\left(r_{j}-\bar{r}_{j}\right)^{2}\right]^{3 / 2}} \\
=\left(\frac{2 \Gamma\left(m_{j}+1 / 2\right)^{3}}{m_{j}^{3 / 2} \Gamma\left(m_{j}\right)^{3}}+\frac{\Gamma\left(m_{j}+3 / 2\right) e^{3 \sigma_{j}^{2} / 4}}{m_{j}^{3 / 2} \Gamma\left(m_{j}\right)}\right. \\
\left.\quad-\frac{3 \Gamma\left(m_{j}+1 / 2\right) e^{\sigma_{j}^{2} / 4}}{\sqrt{m_{j}} \Gamma\left(m_{j}\right)}\right) \\
\quad \times\left(\left(e^{\sigma_{j}^{2} / 4}-\frac{\Gamma\left(m_{j}+1 / 2\right)^{2}}{m_{j} \Gamma(m)^{2}}\right)^{3 / 2}\right)^{-1}, \\
j=1,2,
\end{gathered}
$$

where $\bar{r}_{j}$ is the mean of $r_{j}, j=1,2$. The skewness, $s_{j}$, increases with $\sigma_{j}$. For instance, the skewness oscillates from 3.55 to 12.11 with $m_{j}=1$ for shadowing standard deviations from 6 to 10 $\mathrm{dB}$, respectively. Nevertheless, the skewness decreases slightly with the fading parameter, $m_{j}$. For instance, $s_{j}=3.55$ for $\sigma_{d j}=6 \mathrm{~dB}$ and $m_{j}=1$ and $s_{j}=3.15$ for $\sigma_{d j}=6 \mathrm{~dB}$ and $m_{j}=3$.

Figures 2, 3, and 4 show the bias and the standard deviation of $R$ as a function of the fast fading correlation coefficient,

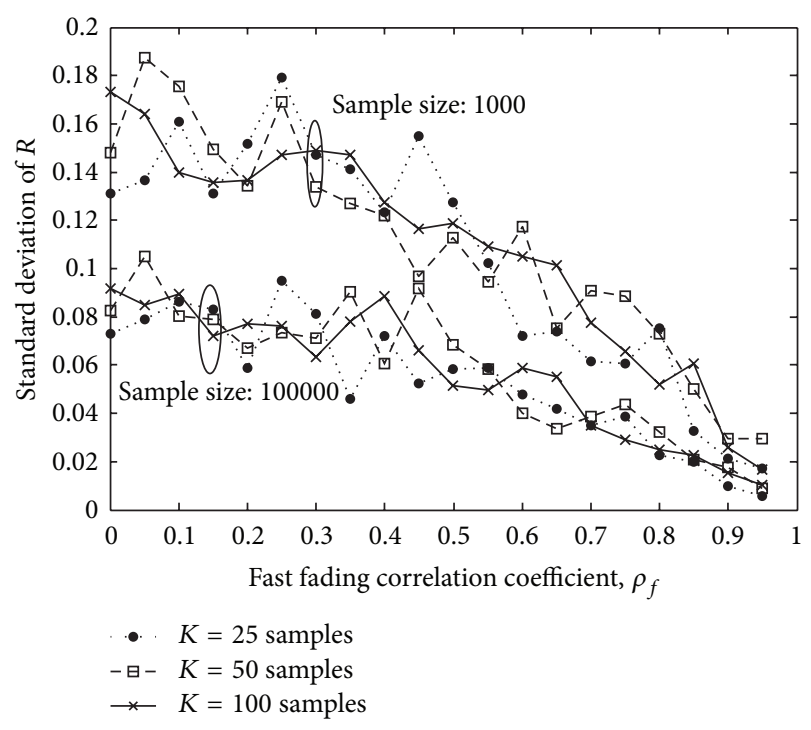

FIGURE 2: Standard deviation of the sample correlation coefficient as a function of the fast fading correlation coefficient for a number of observations $N=1000,100000$ and number of samples $K=$ $25,50,100$. The distribution parameters are $m_{1}=1.2, m_{2}=1.2$; $\sigma_{d_{1}}=\sigma_{d_{2}}=8 \mathrm{~dB}$.

$\rho_{f}$. The results correspond to spatial microdiversity, with $\rho_{s}=$ $1, m=m_{1}=m_{2}$, and $\sigma_{d}=\sigma_{d 1}=\sigma_{d 2}$. In particular, the effect of the sample size, $N$, and the number of samples, $K$, on the variation of the standard deviation is illustrated in Figure 2. In this figure, the parameters of the distribution are $m=m_{1}=m_{2}=1.2$ and $\sigma_{d}=\sigma_{d 1}=\sigma_{d 2}=$ $8 \mathrm{~dB}$. The sample size, which corresponds to the number of observations used in simulations, is $N=1000$ and 100000 , and the number of samples is $K=25,50$, and 100 . Obviously, the higher the number of observations, $N$, the smaller the standard deviation. Also, the standard deviation reduces as the fast fading correlation coefficient, $\rho_{f}$, grows. Nevertheless, the standard deviation of $R$ is scarcely affected by the number of samples calculated; that is, $K$ equals 25,50 , and 100 .

In Figure 3, the effect of both the fading parameter, $m$, and the shadowing standard deviation, $\sigma_{d j}$, on the standard deviation of the sample correlation coefficient is analyzed for $N=100000$ and $K=50$. The standard deviation of the sample correlation coefficient depends strongly on the shadowing standard deviation. Also, it can be observed that the standard deviation of $R$ for $\sigma_{d}=10 \mathrm{~dB}$ decreases as $m$ grows. In general, the standard deviation of $R$ tends to reduce for high correlation coefficient values.

Figure 4 shows the mean of the sample correlation coefficient and the correlation coefficient $\rho$ given by (17), that is, the analytical prediction, as a function of the fast fading correlation coefficient. The simulations are performed for $m=m_{1}=m_{2}=1.2, \sigma_{d}=\sigma_{d_{1}}=\sigma_{d 2}=10 \mathrm{~dB}$ and $m=m_{1}=m_{2}=3.2, \sigma_{d}=\sigma_{d 1}=\sigma_{d 2}=8 \mathrm{~dB}$. The number of observations is $N=100000$ and the number of samples is $K=$ 25,50 , and 100 . Generally speaking, the sample correlation coefficient is slightly positive biased for these values. This bias 


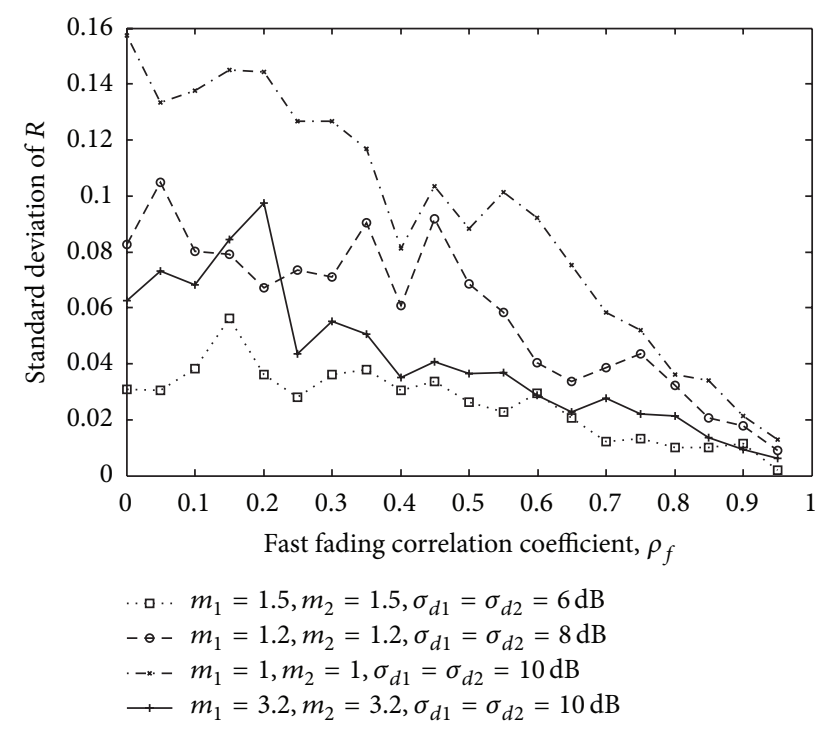

Figure 3: Standard deviation of the sample correlation coefficient as a function of the fast fading correlation coefficient for different parameters of the marginal distributions. The number of observations and number of samples are $N=100000$ and $K=50$, respectively.

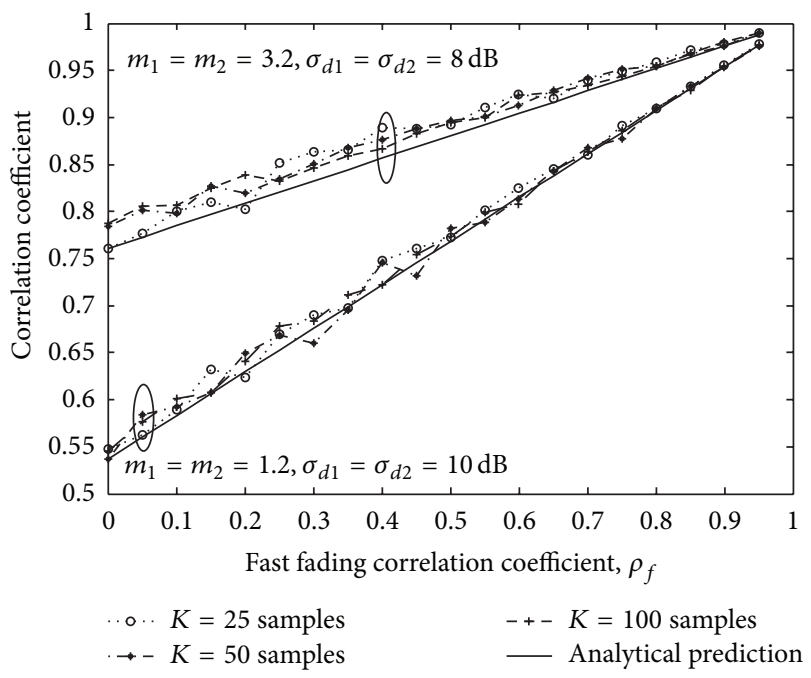

Figure 4: Correlation coefficient and mean of the sample correlation coefficient as a function of the fast fading correlation coefficient for $m_{1}=m_{2}=1.2, \sigma_{d 1}=\sigma_{d 2}=10 \mathrm{~dB}$ and $m_{1}=m_{2}=3.2$, $\sigma_{d 1}=\sigma_{d 2}=8 \mathrm{~dB}$. The correlation coefficient (analytical prediction) is in solid lines and the mean of the sample correlation coefficient in dashed lines. The number of observations is $N=100000$ and the number of samples is $K=25,50,100$.

is higher for small fast fading correlation coefficients and for $m=m_{1}=m_{2}=3.2 ; \sigma_{d}=\sigma_{d 1}=\sigma_{d 2}=8 \mathrm{~dB}$.

In Figure 5, the correlation coefficient given by (16) and the mean of the sample correlation coefficient are plotted as a function of the angle between the MS and the base stations, $\phi$, for $d_{1}=1000 \mathrm{~m}, d_{2}=2000 \mathrm{~m}, d_{c}=300 \mathrm{~m}$, and $\gamma=$ 0.3 . Curves have been drawn for $N=10000,100000$, and 1000000 , and $K=50$. The marginal distributions parameters

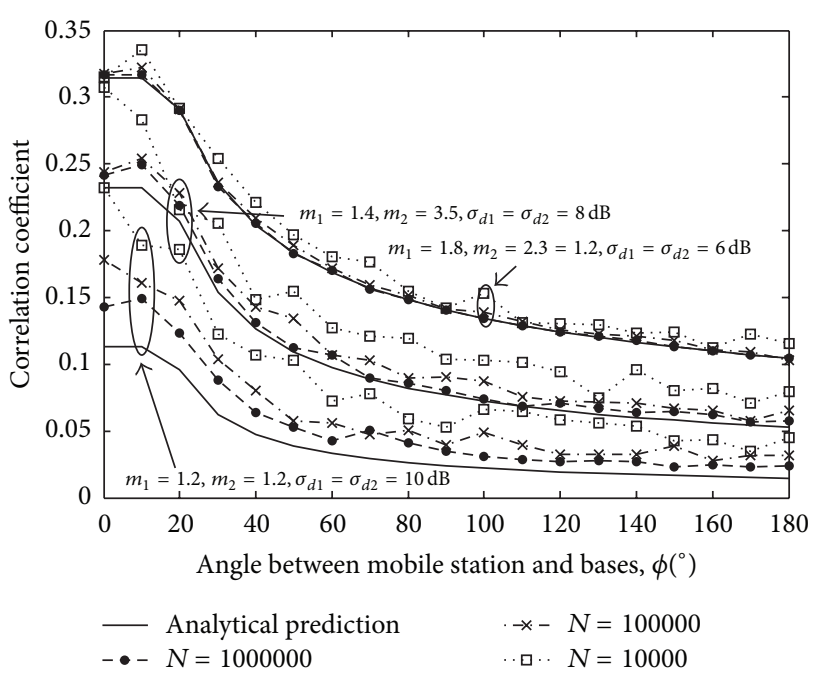

FIGURE 5: Correlation coefficient and mean of the sample correlation coefficient as a function of the angle between the mobile station and the two base stations. The parameters of the shadowing crosscorrelation scenario are $d_{1}=1000 \mathrm{~m}, d_{2}=2000 \mathrm{~m}, d_{c}=300 \mathrm{~m}$, and $\gamma=0.3$. The marginal distributions parameters are $m_{1}=m_{2}=1.2$, $\sigma_{d_{1}}=\sigma_{d_{2}}=10 \mathrm{~dB} ; m_{1}=1.4, m_{2}=3.2, \sigma_{d_{1}}=\sigma_{d_{2}}=8 \mathrm{~dB}$; and $m_{1}=1.8, m_{2}=2.3, \sigma_{d_{1}}=\sigma_{d 2}=6 \mathrm{~dB}$. The correlation coefficient (analytical prediction) is in solid lines and the mean of the sample correlation coefficient in dashed lines. The number of observations is $N=10000,100000,1000000$ and the number of samples is $K=50$.

are $m=m_{1}=m_{2}=1.2, \sigma_{d}=\sigma_{d 1}=\sigma_{d 2}=10 \mathrm{~dB} ; m_{1}=$ 1.4, $m_{2}=3.5, \sigma_{d}=\sigma_{d 1}=\sigma_{d 2}=8 \mathrm{~dB}$; and $m_{1}=1.8, m_{2}=$ 2.3, $\sigma_{d}=\sigma_{d 1}=\sigma_{d 2}=6 \mathrm{~dB}$. It is observed that the sample correlation coefficient is positive biased for such parameter values. This bias is considerably high for a small number of observations, that is, $N=10000$ and $N=100000$, specially for $\phi$ close to $0^{\circ}$. Also, from the results shown in Figure 5, the higher the shadowing standard deviation is, the larger the values of the bias are using the same number of observations and $\phi$. This bias is large for $\sigma_{d}=\sigma_{d 1}=\sigma_{d 2}=10 \mathrm{~dB}$ and low values of $\phi$ even for a high number of observations; for example, the bias is around 0.035 for $m=m_{1}=m_{2}=1.2$, $\phi \leq 10^{\circ}$, and $N=1000000$.

\section{Conclusions}

In this paper, we have analytically derived the PDF of bivariate Nakagami-lognormal distribution with arbitrary distribution parameters of the marginal distributions and arbitrary correlation. Also, the correlation coefficient between the compound signals has been obtained as a function of the distribution parameters and both the fast fading and shadowing correlation coefficients. This correlation coefficient has been particularized for specific scenarios of wireless communications: micro- and macrodiversity. In microdiversity, the correlation coefficient between the composite signals is substantially higher than the fast fading correlation coefficient. Otherwise, the composite correlation coefficients are small (in the examples lower than 0.32) in the macrodiversity scenario. 
Two procedures to generate two correlated Nakagamilognormal variables have been described. In examples of both microdiversity and macrodiversity scenarios, we have evaluated the sample correlation coefficient between the composite Nakagami-lognormal signals, comparing it to the analytical correlation coefficient derived previously. In general, this sample correlation coefficient is positive biased. From those examples, it can be observed that the bias and the standard deviation of the sample correlation coefficient depend substantially on the shadowing standard deviation.

The results reported in this work can be of interest in the assessment of performances (outage probabilities, bit error rates, etc.) in diversity receivers with highly dynamical environments, such as vehicular or indoor wireless channels. In these scenarios, the separation of the fast fading and shadowing processes is cumbersome, and the received signals at each branch of the diversity combiner can be accurately modeled as Nakagami-lognormal composite distributions.

\section{Conflict of Interests}

The authors declare that there is no conflict of interests regarding the publication of this paper.

\section{Acknowledgment}

This work was supported by the Spanish Ministerio de Ciencia e Innovación TEC-2010-20841-C04-1.

\section{References}

[1] J. D. Parsons, Mobile Radio Propagation Channel, John Wiley \& Sons, Chichester, UK, 2nd edition, 2000.

[2] A. F. Molisch, Wireless Communications, John Wiley \& Sons, Chichester, UK, 2nd edition, 2011.

[3] M. Nakagami, "The m-distribution-a general formula of intensity distribution of rapid fading," in Statistical Methods of Radio Wave Propagation, W. G. Hoffman, Ed., pp. 3-35, Pergamon Press, Oxford, UK, 1960.

[4] L. Rubio, J. Reig, and N. Cardona, "Evaluation of Nakagami fading behaviour based on measurements in urban scenarios," International Journal of Electronics and Communications, vol. 61, no. 2, pp. 135-138, 2007.

[5] S. R. Saunders and A. Aragón-Zavala, Antennas and Propagation for Wireless Communication Systems, John Wiley \& Sons, Chichester, UK, 2nd edition, 2007.

[6] H. Suzuki, "A statistical model for urban radio propagation," IEEE Transactions on Communications, vol. 25, no. 7, pp. 673680, 1977.

[7] A. A. Abu-Dayya and N. C. Beaulieu, "Micro- and macrodiversity NCFSK (DPSK) on shadowed Nakagami-fading channels," IEEE Transactions on Communications, vol. 42, no. 9, pp. 26932702, 1994.

[8] M. Ho and G. Stuber, "Co-channel interference of microcellular systems on shadowed Nakagami fading channels," in Proceedings of the 43rd IEEE Vehicular Technology Conference (VTC '93), pp. 568-571, May 1993.

[9] T. T. Tjhung and C. C. Chai, "Fade statistics in Nakagami-lognormal channels," IEEE Transactions on Communications, vol. 47, no. 12, pp. 1769-1772, 1999.
[10] M. D. Yacoub, "The $\alpha-\mu$ distribution: a physical fading model for the Stacy distribution," IEEE Transactions on Vehicular Technology, vol. 56, pp. 122-124, 2007.

[11] P. M. Shankar, "Error rates in generalized shadowed fading channels," Wireless Personal Communications, vol. 28, no. 3, pp. 233-238, 2004.

[12] S. Atapattu, C. Tellambura, and H. Jiang, "A mixture gamma distribution to model the SNR of wireless channels," IEEE Transactions on Wireless Communications, vol. 10, no. 12, pp. 4193-4203, 2011.

[13] J. Reig and L. Rubio, "Estimation of the composite fast fading and shadowing distribution using the log-moments in wireless communications," IEEE Transactions on Wireless Communications, vol. 12, no. 8, pp. 3672-3681, 2013.

[14] S. Mukherjee and D. Avidor, "Effect of microdiversity and correlated macrodiversity on outages in a cellular system," IEEE Transactions on Wireless Communications, vol. 2, no. 1, pp. 5058, 2003.

[15] R. Zhang, J. Wei, D. G. Michelson, and V. C. M. Leung, "Outage probability of MRC diversity over correlated shadowed fading channels," IEEE Wireless Communications Letters, vol. 1, no. 5, pp. 516-519, 2012.

[16] G. C. Alexandropoulos, A. Conti, and P. T. Mathiopoulos, "Adaptive M-QAM systems with diversity in correlated Nakagami-m fading and shadowing," in Proceedings of the 53rd IEEE Global Communications Conference (GLOBECOM '10), pp. 1-5, December 2010.

[17] R. Zhang, J. Wei, and V. C. M. Leung, "Outage probability of composite microscopic and macroscopic diversity over correlated shadowed fading channels," China Communications, vol. 10, pp. 129-142, 2013.

[18] M. Abdel-Hafez and M. Cafak, "Performance analysis of digital cellular radio systems in Nakagami fading and correlated shadowing environment," IEEE Transactions on Vehicular Technology, vol. 48, no. 5, pp. 1381-1391, 1999.

[19] P. M. Shankar, "Macrodiversity and microdiversity in correlated shadowed fading channels," IEEE Transactions on Vehicular Technology, vol. 58, no. 2, pp. 727-732, 2009.

[20] M. Abramowitz, Handbook of Mathematical Functions, with Formulas, Graphs, and Mathematical Tables, Dover, New York, NY, USA, 9th edition, 1972.

[21] M. K. Simon and M. S. Alouini, Digital Communication over Fading Channels, vol. 86, Wiley-IEEE Press, 2nd edition, 2005.

[22] M. D. Mostafa and M. W. Mahmoud, "On the problem of estimation for the bivariate lognormal distribution," Biometrika, vol. 51, pp. 522-527, 1964.

[23] J. Reig, L. Rubio, and N. Cardona, "Bivariate Nakagami$\mathrm{m}$ distribution with arbitrary fading parameters," Electronics Letters, vol. 38, no. 25, pp. 1715-1717, 2002.

[24] C. C. Tan and N. C. Beaulieu, "Infinite series representations of the bivariate Rayleigh and Nakagami-m distributions," IEEE Transactions on Communications, vol. 45, no. 10, pp. 1159-1161, 1997.

[25] D. Lien and N. Balakrishnan, "Moments and properties of multiplicatively constrained bivariate lognormal distribution with applications to futures hedging," Journal of Statistical Planning and Inference, vol. 136, no. 4, pp. 1349-1359, 2006.

[26] K. Zayana and B. Guisnet, "Measurements and modelisation of shadowing cross-correlations between two base-stations," in Proceedings of the IEEE International Conference on Universal Personal Communications (ICUPC '98), vol. 1, pp. 101-105, Florence, Italy, October 1998. 
[27] T. B. Sørensen, "Slow fading cross-correlation against azimuth separation of base stations," Electronics Letters, vol. 35, no. 2, pp. 127-129, 1999.

[28] W. C. Jakes, Microwave Mobile Communications, IEEE Press, Piscataway, NJ, USA, 2nd edition, 1994.

[29] J. Reig, M. A. Martinez-Amoraga, and L. Rubio, "Generation of bivariate Nakagami-m fading envelopes with arbitrary not necessary identical fading parameters," Wireless Communications and Mobile Computing, vol. 7, no. 4, pp. 531-537, 2007.

[30] C. D. Lai, J. C. W. Rayner, and T. P. Hutchinson, "Robustness of the sample correlation-the bivariate lognormal case," Journal of Applied Mathematics and Decision Sciences, vol. 3, no. 1, pp. 7-19, 1999. 

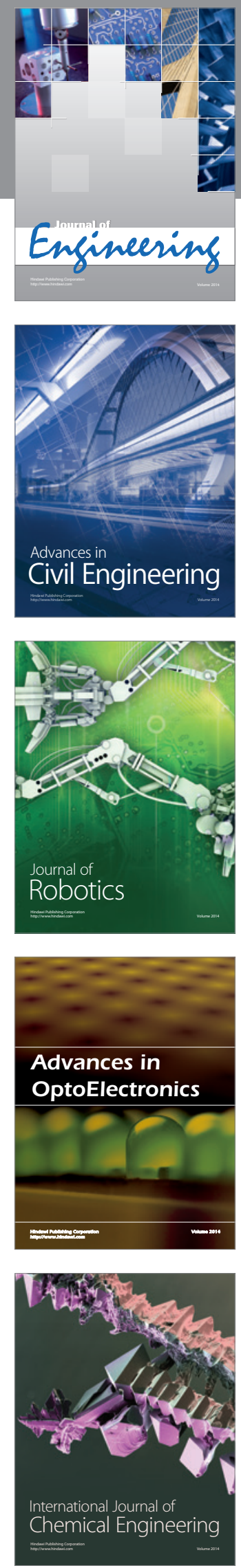

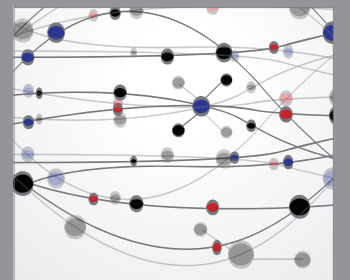

The Scientific World Journal
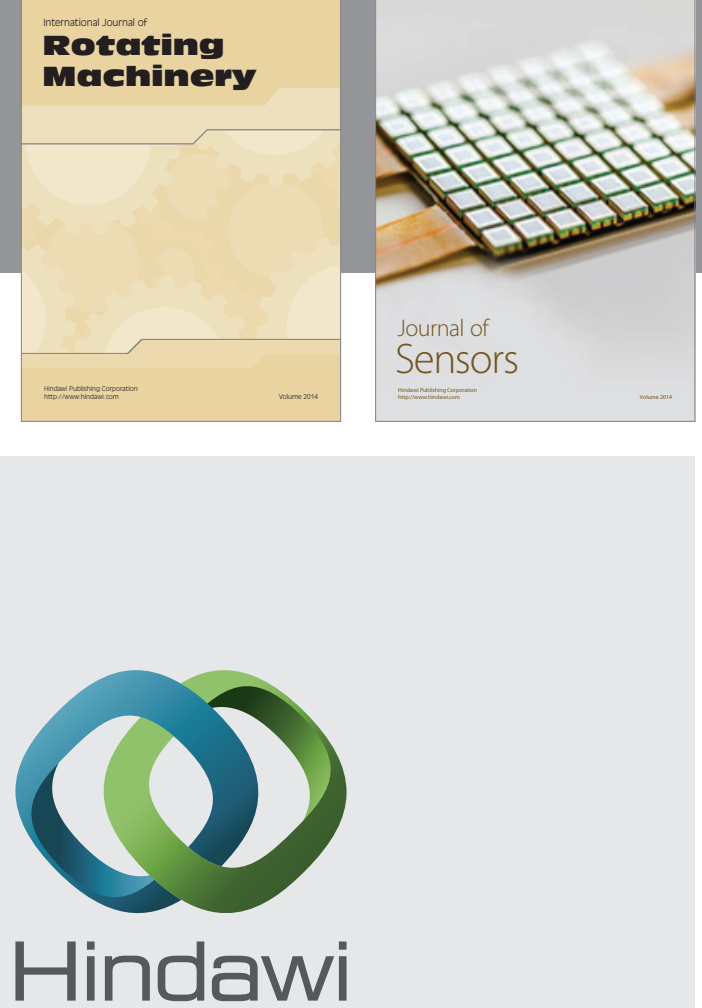

Submit your manuscripts at http://www.hindawi.com
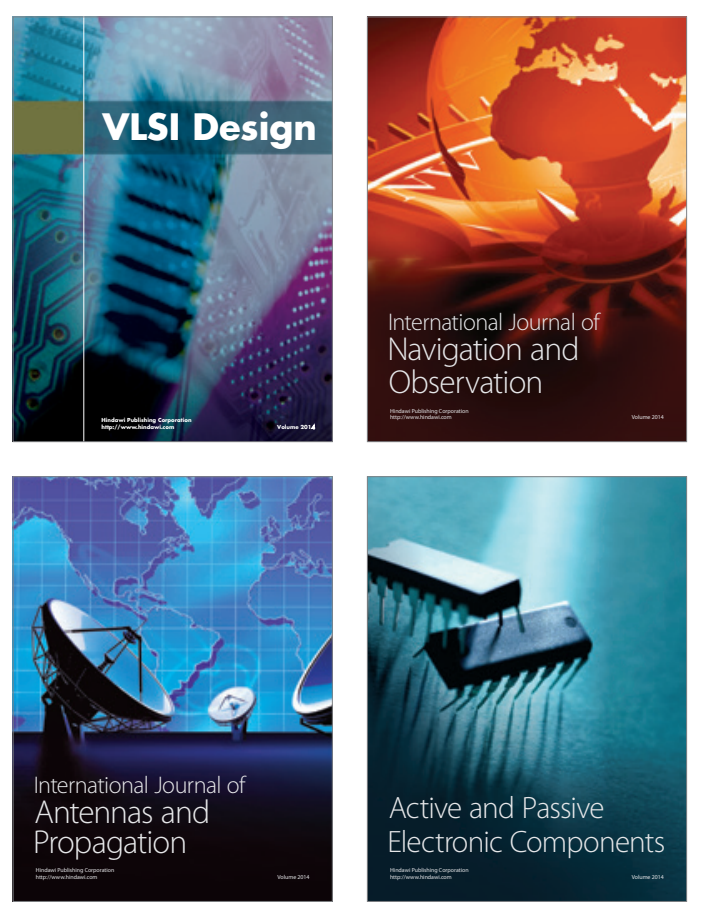
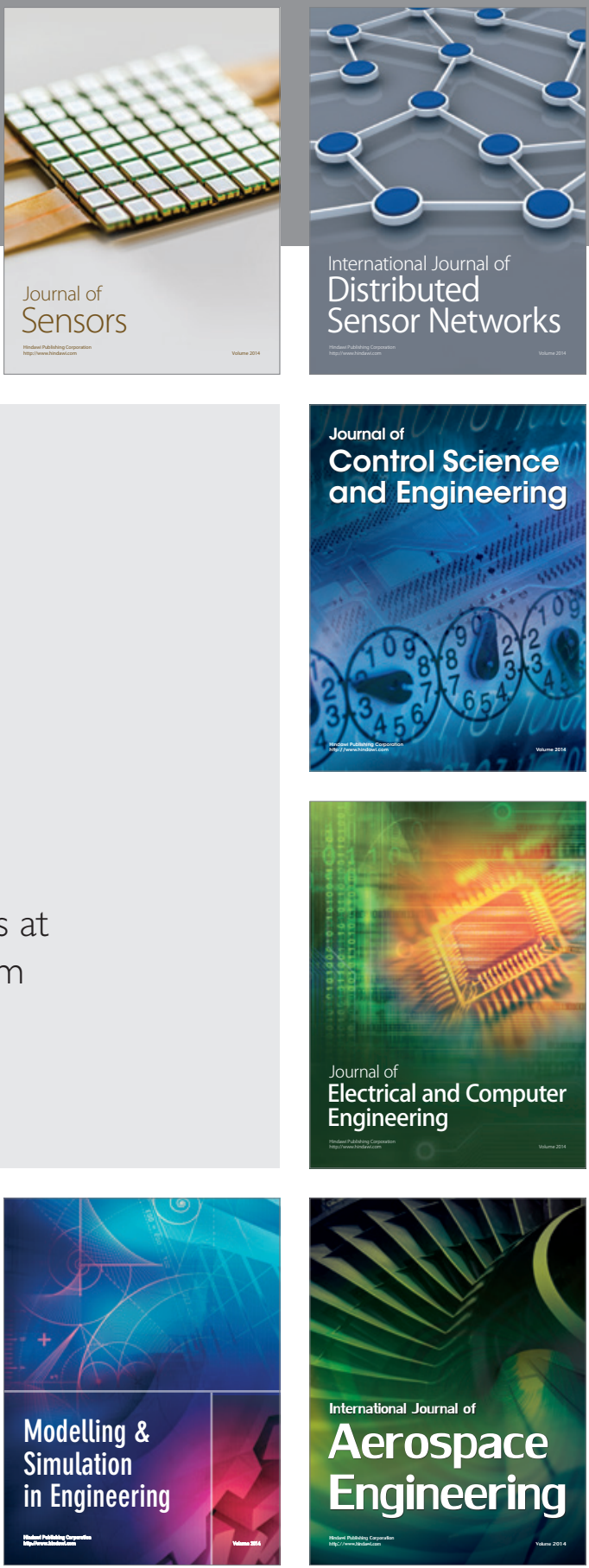

Journal of

Control Science

and Engineering
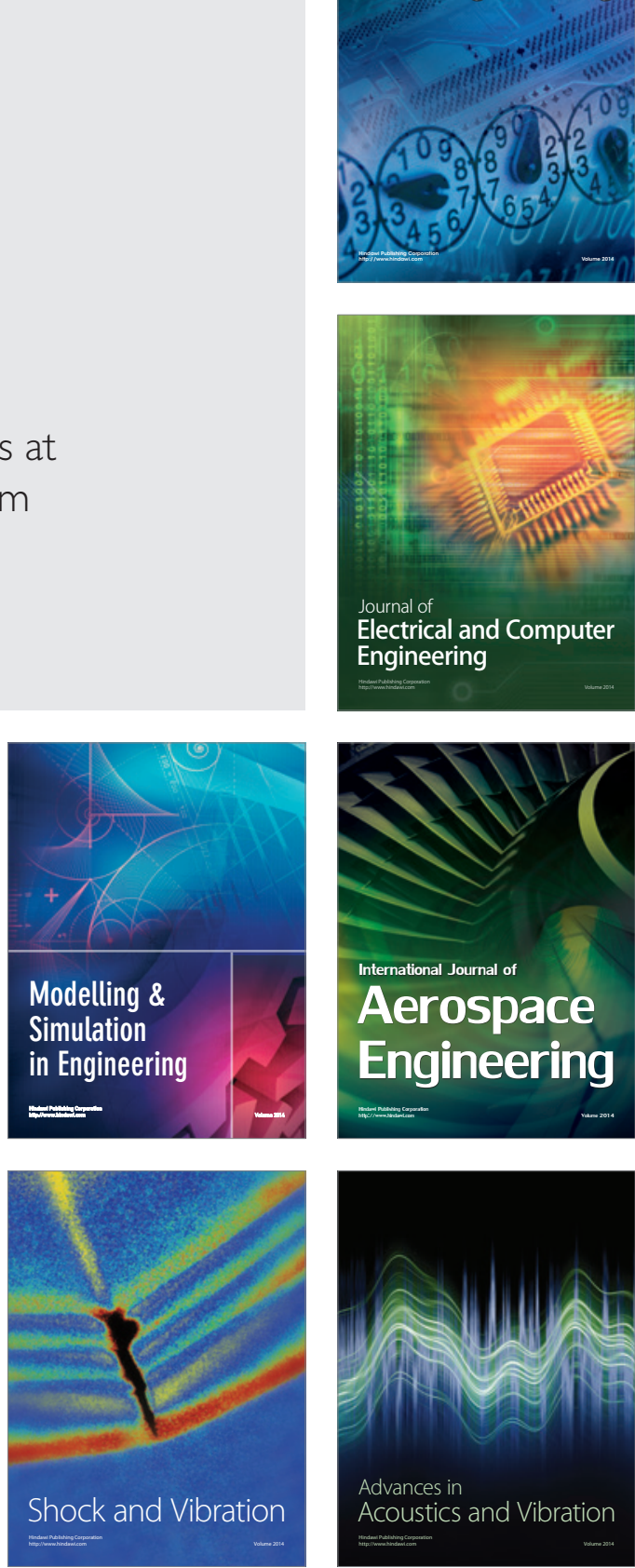